\title{
Clinical and prognostic relevance of CXCL12 expression in acute myeloid leukemia
}

\author{
Shi-sen Wang ${ }^{\text {Equal first author, } 1}$, Zi-jun Xu ${ }^{\text {Equal first author, 2, } 3}$, Ye Jin ${ }^{1,3}$, Ji-chun Ma ${ }^{2,3}$, Pei-hui Xia ${ }^{2,3}$, Xiangmei Wen ${ }^{2,3}$, Zhen-wei \\ Mao ${ }^{\text {Corresp., } 2 \text {, Jiang Lin }}{ }^{2,3}$, Jun Qian ${ }^{\text {Corresp. 1, } 3}$ \\ 1 Department of Hematology, Affiliated People's Hospital of Jiangsu University, Zhenjiang, Jiangsu, China \\ 2 Laboratory Center, Affiliated People's Hospital of Jiangsu University, Zhenjiang, Jiangsu, China \\ 3 Zhenjiang Clinical Research Center of Hematology, Zhenjiang, Jiangsu, China \\ Corresponding Authors: Zhen-wei Mao, Jun Qian \\ Email address: maopen365@163.com, qianjun@jskfhn.org.cn
}

Background. Accumulating studies have been made to understand the association between CXC chemokine ligand-12 (CXCL12)/CXC chemokine receptor 4 (CXCR4) and acute myeloid leukemia (AML). However, large-scale data analysis of potential relationship between CXCL12 and AML remains insufficient. Methods. We collected abundant CXCL12 expression data and AML samples from several publicly available datasets. CIBERSORT algorithm was used to quantify immune cell fractions and the online website of STRING was utilized for gene ontology (GO) enrichment and Kyoto encyclopedia of genes and genomes (KEGG) analysis. The statistical analysis and graphical work were mainly performed via the R software. Results. CXCL12 expression was extremely down-regulated in AML. Clinically, low CXCL12 expression was correlated with higher white blood cells (WBCs) $(P<0.0001)$, more blasts in bone marrow $(B M)(P<0.001)$ and peripheral blood (PB) $(P<0.0001)$, FLT3-internal tandem duplications (FLT3-ITD) $(P=0.010)$ and NPM1 mutations $(P=0.015)$. More importantly, reduced $C X C L 12$ expression predicted worse overall survival (OS) and event-free survival (EFS) in all AML, non-M3-AML, and cytogenetically normal (CN)-AML patients in three independent cohorts. As for immune cell infiltration, high CXCL12 expressed groups tended to harbor more memory B cells and plasma cells infiltration while low CXCL12 expressed groups exhibited more eosinophils infiltration. GO enrichment and KEGG pathways analysis revealed the potential biological progress the gene participating in. Conclusions. CXCL12 is significantly down-regulated in AML and low CXCL12 expression is an independent and poor predictor of AML prognosis. CXCL12 expression level correlates with clinical and immune characteristics of AML, which could provide potential assistance for treatment. Prospective studies are needed to further validate the impact of CXCL12 expression before routine clinical application in AML. 


\section{Clinical and prognostic relevance of CXCL12}

\section{2 expression in acute myeloid leukemia}

3

4

5 6 7

8

9 Jiangsu, P.R. China

10

11 China

Corresponding Author:

Jun Qian ${ }^{1,3}$

Zhen-wei Mao ${ }^{2}$

Shi-sen Wang ${ }^{1 \dagger}$, Zi-jun $\mathrm{Xu}^{2,3 \dagger}$, Ye Jinn ${ }^{1,3}$, Ji-chun Ma ${ }^{2,3}$, Pei-hui Xia ${ }^{2,3}$, Xiang-mei Wen ${ }^{2,3}$, Zhenwei $\mathrm{Mao}^{2}$, Jiang $\operatorname{Lin}^{2,3}$, Jun Qian ${ }^{1,3}$

${ }^{1}$ Department of Hematology, Affiliated People's Hospital of Jiangsu University, Zhenjiang, ${ }^{2}$ Laboratory Center, Affiliated People's Hospital of Jiangsu University, Zhenjiang, Jiangsu, P.R.

${ }^{3}$ Zhenjiang Clinical Research Center of Hematology, Zhenjiang, Jiangsu, P.R. China

${ }^{\dagger}$ Shi-sen Wang and Zi-jun Xu should be considered joint first author

8 Dianli Rd., Zhenjiang, Jiangsu, 212002, P.R. China

Email address: qianjun@jskfhn.org.cn

8 Dianli Rd., Zhenjiang, Jiangsu, 212002, P.R. China

Email address: maopen365@163.com 


\section{Abstract}

\section{Introduction}

Background. Accumulating studies have been made to understand the association between $C X C$ chemokine ligand-12 (CXCL12)/CXC chemokine receptor 4 (CXCR4) and acute myeloid leukemia (AML). However, large-scale data analysis of potential relationship between CXCL12 and AML remains insufficient.

Methods. We collected abundant CXCL12 expression data and AML samples from several publicly available datasets. CIBERSORT algorithm was used to quantify immune cell fractions and the online website of STRING was utilized for gene ontology (GO) enrichment and Kyoto encyclopedia of genes and genomes (KEGG) analysis. The statistical analysis and graphical work were mainly performed via the R software.

Results. CXCL12 expression was extremely down-regulated in AML. Clinically, low CXCL12 expression was correlated with higher white blood cells (WBCs) $(P<0.0001)$, more blasts in bone marrow $(\mathrm{BM})(P<0.001)$ and peripheral blood $(\mathrm{PB})(P<0.0001)$, FLT3-internal tandem duplications $(F L T 3-\mathrm{ITD})(P=0.010)$ and NPM1 mutations $(P=0.015)$. More importantly, reduced CXCL12 expression predicted worse overall survival (OS) and event-free survival (EFS) in all AML, non-M3-AML, and cytogenetically normal (CN)-AML patients in three independent cohorts. As for immune cell infiltration, high CXCL12 expressed groups tended to harbor more memory B cells and plasma cells infiltration while low CXCL12 expressed groups exhibited more eosinophils infiltration. GO enrichment and KEGG pathways analysis revealed the potential biological progress the gene participating in.

Conclusions. CXCL12 is significantly down-regulated in AML and low CXCL12 expression is an independent and poor predictor of AML prognosis. CXCL12 expression level correlates with clinical and immune characteristics of AML, which could provide potential assistance for treatment. Prospective studies are needed to further validate the impact of $C X C L 12$ expression before routine clinical application in AML.

Acute myeloid leukemia (AML) represents a heterogeneous disease characterizing by complex molecular signatures, complicated pathogeny and aggressive progression, as well as a poor clinical outcome (Döhner et al. 2015). Bone marrow (BM) microenvironment contributes significantly to leukemogenesis and leukemia progression (Ayala et al. 2009; Meads et al. 2008). 
69 Most adult patients are still terribly suffering from AML, even though tremendous advancements 70 such as novel chemotherapy drugs and hematopoietic stem cell transplantation (HSCT) have 71 been achieved recently (Döhner et al. 2017; Döhner et al. 2015). Until now, chromosome 72 aberration analysis remains the main method for diagnosis, classification, prognosis, and guiding 73 clinical therapy while $40-50 \%$ of patients are lack representative chromosome aberrations 74 (Mrózek et al. 2004). So more specific and accurate biomarkers are necessary to be discovered 75

76

77 78 79 80 81 for AML. In recent years, immunophenotype and cytochemistry analysis are found valuable in the diagnosis of AML, and immunophenotyping surface type molecules like CD123, CD45, CD34, CD38 have been reported to help confirm the diagnosis of AML (Prada-Arismendy et al. 2017). Additionally, it is now evident that oncogene mutations affect the progression of AML and some of these mutated genes are FLT3, NPM1, and CEBPA (Grove \& Vassiliou 2014; Meyer \& Levine 2014). Unfortunately, despite extensive researches that have been carried out to find prognostic biomarkers, AML is still a tough disease with a variable prognosis and poor survival rate, with 5-year overall survival less than 50\% and even lower in the elderly (Kell 2004). Considering all the above, further studies are essential for a better understanding of how various factors contribute to the disease progression.

Chemokines and their receptors mediate crucial biological functions of tumor progression including leukocyte recruitment and function, cellular senescence, tumor cell proliferation and survival, and invasion and metastasis (Mantovani et al. 2010). $C X C$ chemokine ligand-12 (CXCL12), also known as stromal cell-derived factor-1 (SDF-1), is a $C X C$ chemokine that belongs to the large family of chemotactic cytokines. The protein, functioning through its receptor $C X C$ chemokine receptor $4(C X C R 4)$, plays an important role in hematopoietic cell development, immune system foundation, and retention of hematopoietic stem and progenitor cells in the BM microenvironment (Janssens et al. 2018). CXCL12 expression has been found to be related to at least 23 different types of tumors (Balkwill 2004) and the gene's critical influences on solid tumors have been illustrated in prostate cancer (Taichman et al. 2002), nonsmall cell lung cancer (Phillips et al. 2003), renal cell carcinoma (Pan et al. 2006) and so on. As for hematological malignancies, CXCL12 is reported to be overexpressed in chronic lymphocytic leukemia (CLL) (Möhle et al. 1999), as well as in childhood acute lymphoblastic leukemia (Crazzolara et al. 2001). Also, Spoo AC et al. have proved that elevated CXCR4 expression is relevant to inferior prognosis of AML in adult patients (Konoplev et al. 2007; Rombouts et al. 2004; Spoo et al. 2007). All these findings may contribute to a better understanding of CXCL12's potential mechanism and clinical relevance in both solid tumors and blood cancers.

Despite enormous progress having been made to understand the relationship between $C X C R 4$ and AML, studies concentrating on CXCL12 and AML remain insufficient, and large-scale analysis of the potential association between the gene and AML hasn't been performed before. 
105 Herein, we adopted similar methods of our previous research (Xu et al. 2019) and made

106

107

108

109

110

111

112

113

114

115

116

117

118

119

120

121

122

123

124

125

126

127

128

129

130

131

132

133

134

135

136

137

138

139 comprehensive analyses of the gene's possible interaction with AML using public datasets, trying to reveal the implication of $C X C L 12$ expression in AML and providing potential treatment strategies for the clinic.

\section{Materials \& Methods}

\section{Patients and Database}

We used six publicly available datasets from Gene Expression Omnibus (GEO, http://www.ncbi.nlm.nih.gov/geo/) and The Cancer Genome Atlas (TCGA, https://cancergenome.nih.gov/) in this study. Of these datasets, three of them consist of expression data for bulk primary AML samples (the TCGA dataset, GSE6891, and GSE10358), two involve expression data for healthy and AML bone marrow samples (GSE30029 and GSE63270) and one includes expression data for major hematopoietic lineages during the differentiation (GSE42519), respectively. Expression data for FAB AML subtypes and patient characteristics were acquired from TCGA sequencing data. Normal mRNA expression data from Genotype-Tissue Expression (GTEx) database and pan-cancer cell line sequencing data from Broad Institute Cancer Cell Line Encyclopedia (CCLE) database were extracted through their portal websites for analysis.

\section{Estimation of immune cell fractions}

CIBERSORT algorithm (https://cibersort.stanford.edu/index.php) was used to quantify immune cell fractions according to the expression pattern of CXCL12. More detailed information for this method is available in Newman AM et al.'s introduction (Newman et al. 2015). Immune cells calculated in this study contains B cells (including B cells memory and B cells naive), $\mathrm{T}$ cells (including CD8+ T cells, CD4+ naive T cells, CD4+ memory resting T cells, CD4+ memory activated $\mathrm{T}$ cells, follicular helper $\mathrm{T}$ cells, regulatory $\mathrm{T}$ cells and $\mathrm{T}$ cells gamma delta), macrophage (including M0 macrophage, M1 macrophage, and M2 macrophage), NK cells (including NK cells resting and NK cells activated), dendritic cells (including dendritic cells resting and dendritic cells activated), mast cells (including mast cells resting and mast cells activated), plasma cells, monocyte, eosinophils, and neutrophils. Multiple immune infiltration deconvolution methods including TIMER, quanTIseq, xCell, MCP-counter, and EPIC were achieved from their websites.

\section{Statistical analysis and bioinformatics}

Low and high CXCL12 expressers were discriminated against based on the median expression level of the gene. Analyses of patient characteristics and survival were described previously (Xu et al. 2019). Differential expression was analyzed using 'limma' or 'edgeR' R package. 
140 The Pearson Correlation coefficient (denoted as Cor) was calculated to assess whether there was

141 a correlation between cell infiltration and CXCL12 expression. Moreover, 'ggplot2' and 'ggpubr'

142 R packages were applied for visualizing the results of data analysis. The single-cell correlation

143 analysis of CXCL12 was conducted using single-cell data sequenced by van Galen, P. et al (van

144 Galen et al. 2019), and the published scRNA-seq dataset was downloaded from

145 GEO: GSE116256. And gene ontology (GO) enrichment and Kyoto encyclopedia of genes and 146 genomes (KEGG) analysis were conducted using the online website of STRING (http://string-

$147 \mathrm{db}$.org). One-way ANOVA was used to test for differences among at least three groups and the

148 Wilcoxon test was used to determine differences in each two-group comparison. All statistical

149 analyses and figure plotting were performed using the R software version 3.5.3 (https://www.r-

150 project.org/). All differences were considered statistically significant at the level of $P<0.05$.

151

152 Results

153 A systematic overview of differential genes and cytokines in AML

154 Initially, we systematically analyzed a total of 261 cytokine and cytokine receptor genes

155 (Supplementary Data 1) in AML patients and normal controls utilizing GSE30029, and all genes

156 were screened in Supplementary Data 2 with the difference considered significant if adjusted $p$ -

157 value $<0.05$ and $|\operatorname{logFC}|>0$. As obviously displayed in Fig. 1A, the CXCL12 gene was one of the most significantly different genes between AML patients and normal controls. Then, we further verified the differences in GSE63270 (Fig. 1B, Supplementary Data 3). The top 50 genes with significant differences were extracted from two analytic results respectively and then intersected. The common 11 genes included CXCL12, TNFRSF10B, IL1RAP, IL12RB1, IL3RA, CSF2RA, EPOR, TNFSF14, IL18BP, TNFRSF4, and IL2RG. Furthermore, considering the essential functions of $C X C L 12$ in AML, we ultimately decided to study CXCL12 in detail.

\section{CXCL12 expression is different among normal tissues and various carcinomas}

165

To explore how $C X C L 12$ is expressed across various tissues in healthy people, we analyzed the mRNA expression status utilizing the GTEx database (Fig. 2A, Supplementary Data 4). Apparently, CXCL12 expression was extremely low in blood and bone marrow while it was much higher among other normal tissues. It was also worth mentioning that the brain, stomach, and testis showed a relatively low level of CXCL12 expression compared with other tissues. More importantly, when analyzing different cancer cell lines (Supplementary Data 5), we found CXCL12 was lowly expressed among most hematopoietic neoplasms, especially in AML and chronic myelocytic leukemia (CML). Interestingly, cervix cancer cells showed exclusively lower CXCL12 expression levels than AML and CML, as shown in Fig. 2B. 
175 Considering our purpose, we studied the CXCL12 expression difference between healthy people 176 and AML patients. A significant decrease of CXCL12 expression was identified in AML cells 177 (GSE30029, $\mathrm{n}=90)$ in comparison to normal cells (GSE30029, $\mathrm{n}=31)(P=3.2 \mathrm{e}-16$, Fig. $1783 \mathrm{~A})$. We detected a similar result in the GSE63270 cohort $\left(\mathrm{n}_{\mathrm{AML}}=62, \mathrm{n}_{\text {normal }}=42, P=\right.$ 179 7.4e-06), as indicated in Fig. 3B. Additionally, we analyzed CXCL12 expression difference in 180 eight FAB AML subtypes from M0 to M7 (Fig. S1) and CXCL12 transcription during

181

182

183

184

185

186

187

188

189

190

191

192

193

194

195

196

197

198

199

200

201

202

203

204

205

206

207

208

209

210 hematopoiesis differentiation (Fig. S2), although the difference was of no statistical significance.

For further exploration, we delineated CXCL12 expression patterns in several hematological cells including hematopoietic stem cells (HSCs), multipotent progenitor cells (MPPs), granulomonocyte progenitor cells (GMPs), common myeloid progenitor cells (CMPs), megakaryocyteerythroid progenitor cells (MEPs), monocytes, and erythrocytes using the single-cell data sequenced by van Galen, P. et al (van Galen et al. 2019), as shown in Fig. S3A. Interestingly, unlike the former result, MPPs and MEPs showed exclusively high expression of $C X C L 12$. Also, we studied $C X C L 12$ and CXCR4 expression status in the single-cell sequenced data and found CXCR4 expression was abundant in single cells from all patients while CXCL12 was rarely expressed (Fig. S3B).

\section{Clinical features and molecular insights of $C X C L 12$}

The patient characteristics with respect to $C X C L 12$ expression from TCGA were summarized (Table 1). Low CXCL12 expressed patients tended to have higher white blood cell (WBC) counts $(P<0.0001)$ and more percentages of blasts in BM $(P<0.001)$ and peripheral blood $(\mathrm{PB})$

$(P<0.0001)$. With regard to frequently mutated genes in AML, low CXCL12 expressers were more likely to have FLT3-internal tandem duplications (FLT3-ITD) $(P=0.010)$ and NPM1 mutations $(P=0.015)$ and less likely to have mutated TP53 $(P=0.046)$ than those with high CXCL12 expression.

\section{Decreased $C X C L 12$ expression predicts adverse prognosis}

Survival analyses were further performed using three independent cohorts (TCGA, GSE6891, and GSE10358), and all cohorts were divided base on optional cut-off values determined by the x-tile method. In the TCGA whole-AML cohort, $C X C L 12^{\text {low }}$ patients showed significantly decreased overall survival $(\mathrm{OS})(P=0.013$, Fig. 4A) and event-free survival (EFS) $(P=$ 0.0001 , Fig. 4D). The impact of $C X C L 12$ expression on outcome was also observed in the nonM3-AML (OS, $P=0.002$, Fig. 4B; EFS, $P=0.0007$, Fig. 4E) and cytogenetically normal (CN)-AML (OS, $P=0.002$, Fig. 4C; EFS, $P=0.002$, Fig. 4F) subgroups. This finding was further validated using GSE6891 (Fig. S4) and GSE10358 (Fig. S5) and the results were identical to the former.

The potential relationship between low CXCL12 expression and the existence of FLT3-ITD and NPM1 mutations led us to further explore the additional prognostic value of the gene in more 
211 subdivided groups. As expected, low CXCL12 expression patients showed significantly inferior 212 survival probability in the FLT3-ITD absent (Fig. 5A, $P=0.003$ ) and NPMI mutated (Fig. 5B, $213 P=0.068$, a trend for significance actually) patients, which could provide more subdivision 214 proofs for AML based on the dichotomous stratification of CXCL12 expression.

215 Multivariate analysis of $C X C L 12$ expression for OS in the TCGA cohorts

216 To evaluate whether there were potential interrelationships between CXCL12 expression and 217 known clinical factors, we first conducted a univariate analysis in the TCGA dataset and then

218 covered variables with a univariable $P \leqslant 0.20$ in the multivariate Cox proportional hazard

219

220

221

222

223

224

225

226

227

228

229

230

231

232

233

234

235

236

237

238

239

240

241

242

243

244

245

model. The multivariate analysis result of CXCL12 expression for OS was displayed in Table 2. Low $C X C L 12$ expression maintained a high hazard rate for whole-AML patients (HR $=2.25$, 95\% CI 1.51-3.35, $P<0.0001)$, after adjusting age $(P<0.0001)$, WBC count $(P<$ $0.0001)$, cytogenetic risk $(P=0.002)$ and mutation status of TP53 ( $P=0.004), R U N X 1$ ( $P$ $=0.023)$ and DNMT3A $(P=0.012)$. The predictive significance also applied to CN-AML patients $(\mathrm{HR}=2.14,95 \%$ CI $1.15-3.97, P=0.016)$, as indicated.

\section{Profile of relationship between $C X C L 12$ expression and immune cells infiltration}

The landscape of the relationship between CXCL12 expression and immune cell infiltration was systematically evaluated by the CIBERSORT algorithm (Fig. 6). We found that B cells memory, plasma cells, $T$ cells regulatory (Tregs), $T$ cells gamma delta, NK cells activated, mast cells activated, and eosinophils were significantly changed between high CXCL12 and low CXCL12 groups, while $\mathrm{B}$ cell naive, $\mathrm{T}$ cells $\mathrm{CD} 8, \mathrm{~T}$ cells $\mathrm{CD} 4$ naive, $\mathrm{T}$ cells $\mathrm{CD} 4$ memory resting, $\mathrm{T}$ cells CD4 memory activated, $\mathrm{T}$ cell follicular helper, NK cells resting, monocyte, macrophages M0, macrophages M1, macrophages M2, dendritic cells resting, dendritic cells activated, mast cells resting, and neutrophils were not obviously altered between groups. More specifically, high CXCL12 expressed groups tended to harbor more memory B cells and plasma cells infiltration while low $C X C L 12$ expressed groups exhibited more eosinophils infiltration. Less importantly, $\mathrm{T}$ cells regulatory (Tregs), T cells gamma delta, and NK cells were rarely infiltrated in both high and low CXCL12 expressed groups.

To verify the signatures of immune infiltration, we adopted multiple deconvolution methods including TIMER, quanTIseq, xCell, MCP-counter, and EPIC to cross-check the analytic results of CIBERSORT. Then, we made a Pearson correlation analysis between all infiltration results and CXCL12 expression, and totally 16 significantly correlated results were displayed in Fig. S6. Plasma cells (xCell, Cor $=0.273, P<0.001$ ) and B cells (quanTIseq, Cor $=0.246, P<$ 0.001 ; EPIC, Cor $=0.198, P=0.007$; MCP-counter, Cor $=0.145, P=0.050$ ) had a positive correlation with $C X C L 12$ expression.

\section{GO analysis and KEGG pathways analysis of CXCL12}

Peer) reviewing PDF | (2021:03:59363:1:1:NEW 27 Jun 2021) 
$246 \mathrm{GO}$ analyses of CXCL12 for biological process (BP), cellular component (CC) and molecular 247 function (MF) were performed on the online website of STRING (http://string-db.org) (Fig. 7), 248 using genes differentially expressed between high and low CXCL12 expressers $(|\log \mathrm{FC}|>1.5$, 249 adjusted $p$-value $<0.05$, Supplementary Data 6). GO term annotation showed that CXCL12 250 was correlated with anatomical structure morphogenesis, extracellular structure organization, 251 animal organ morphogenesis, biological adhesion, system development, multicellular organismal 252 253 process, animal organ development, cell adhesion, extracellular matrix organization, and multicellular organism development (BP); extracellular region, extracellular space, extracellular

254

255

256

257

258

259

260

261

262

263

264

\section{Discussion}

266

267

268

269

270

271

272

273

274

275

276

277

278

279 matrix, intrinsic component of plasma membrane, integral component of plasma membrane, cell periphery, plasma membrane, collagen-containing extracellular matrix, basement membrane, and collagen trimer (CC); binding, growth factor binding, signaling receptor binding, calcium ion binding, cell adhesion molecule binding, glycosaminoglycan binding, chemokine activity, heparin binding, receptor ligand activity, and receptor regulator activity (MF) (Fig. 7, Supplementary Data 7). GO analysis showed significantly high enrichment in anatomical structure morphogenesis for biological process, extracellular region for cellular component, and binding for molecular function. Moreover, $C X C L 12$ was involved directly or indirectly in the protein digestion and absorption pathway according to the KEGG pathways analysis (Fig. 7, Supplementary Data 7).

Our study showed CXCL12 expression was extremely low in blood and bone marrow compared with other normal tissues and also downgraded in hematological neoplasms among pancarcinomas. For our purpose, we then validated the expression differences between AML and their normal contrasts in two independent cohorts and found a consistent result. Herein, we concluded that $C X C L 12$ was notably down-regulated in AML. Hitherto, there hasn't been enough researches to investigate $C X C L 12$ expression in AML, although its receptor $C X C R 4$ has been demonstrated to be overexpressed in AML (Rombouts et al. 2004). To our knowledge, CXCL12 plays a prominent role in the interaction between AML cells and the BM microenvironment, and the CXCL12/CXCR4-axis is importantly involved in the migration, infiltration, and overproliferation of AML cells (Ladikou et al. 2020).

The clinical implications of $C X C L 12$ were further investigated. $C X C L 12^{\text {low }}$ patients tended to have higher WBC counts and more percentages of blasts, implying more active proliferation of CXCL12-reduced AML cells. Moreover, CXCL12 $2^{\text {low }}$ patients were more likely to harbor FLTITD and NPMI mutations and less often to have TP53 mutation. 
280

281

282

283

284

285

286

287

288

289

290

291

292

293

294

295

296

297

298

299

300

301

302

303

304

305

306

307

308

309

310

311

312

313

314

315

Concerning prognostic relevance, we identified and validated low CXCL12 expression as a prognostic indicator for inferior OS and EFS in all AML, non-M3-AML, and CN-AML patients. In fact, high CXCR4 expression level has been associated with inferior prognosis of AML (Burger \& Bürkle 2007; Spoo et al. 2007) and CXCR4 antagonists such as AMD3100 have been proven to enhance chemosensitization of AML (Nervi et al. 2009). However, it was interesting to discover the predictive value of $C X C L 12$ for AML prognosis exactly opposite to CXCR4, which was beyond our expectation. Previously, Petit I et al. demonstrated that up-regulation of $C X C R 4$ could serve to increase the sensitivity of cells to lower CXCL12 signals (Petit et al. 2002), and Mandawat A et al. found that exposure to CXCL12 caused the lowering of the surface expression of CXCR4 on AML cells (Mandawat et al. 2010), which might provide an explanation for this phenomenon. Moreover, we pointed out that FLT3-ITD absent patients and NPM1 mutated patients could be further subdivided into high-risk groups (with CXCL12 down-regulation) and low-risk groups (with CXCL12 up-regulation) respectively based on the expression level of CXCL12.

GO analysis helped us understand the concrete functions of CXCL12 and the result showed that the gene dynamically participated in anatomical structure morphogenesis for biological process, extracellular region for cellular component, and binding for molecular function. It was also reasonable to extrapolate protein digestion and absorption pathway was curbed molecularly in some way during the development of AML. It has been widely evidenced that CXCL12 plays an essential role in stem cell anchorage to the BM microenvironment and reduction of CXCL12 concentrations within the BM may interfere with retention and facilitate the egress of cells (Petit et al. 2002), which could promote the progress of AML. As previously demonstrated, CXCL12 promoted glycolytic reprogramming in AML cells (Braun et al. 2016) whereas the malignant cells could benefit from aerobic glycometabolism, which endowed them with better capacity for cell proliferation, immune evasion, and chemotherapeutics resistance (Vander Heiden et al. 2009). Low CXCL12 expression restrained glycolytic reprogramming and indirectly promoted aerobic glycometabolism might become one of the possible mechanisms for low CXCL12 expression inducing poor AML prognosis. When analyzing the relationship between CXCL12 and immune cell infiltration, we found high CXCL12 expressed groups tended to harbor more memory B cells and plasma cells infiltration while low CXCL12 expressed groups exhibited more eosinophils infiltration. However, the pathological role of CXCL12 in AML is still largely unknown and needs to be elucidated in future studies.

Although we had been committing ourselves to make the research as comprehensive and credible as possible, there were still several drawbacks that couldn't be ignored. Firstly, the collections were entirely acquired from the public database due to the quantity of clinical data was too limited to conduct such a large-scale analysis. We attempted to mend it by analyzing 
316 multi-group independent datasets to guarantee our results convincing. And secondly, we did not 317 carry out subsequent experiments to authenticate our results, so more laboratory proofs such as 318 protein level verification are required to be offered for our research.

\section{Conclusions}

321 In summary, we carry out a large-scale data analysis of the potential relationship between 322 CXCL12 and AML for the first time. CXCL12 is significantly down-regulated in AML and low 323 CXCL12 expression is an independent and poor predictor of clinical outcome of AML. The gene

324

325

326

327 328

329

330

331

332

333

334

335

336

337

338

339

340

341

342

343

344

345

346

347

348

349

350

351

could provide additional prognostic value in FLT3-ITD absent or NPM1 mutated AML patients.

Furthermore, our research replenishes the understanding of the immune and clinical association between CXCL12 and AML, which could help clinicians to explore new targeted drugs and immunotherapy for AML. Finally, further experiments are warranted to support our findings.

\section{Acknowledgements}

None.

\section{References}

Ayala F, Dewar R, Kieran M, and Kalluri R. 2009. Contribution of bone microenvironment to leukemogenesis and leukemia progression. Leukemia 23:2233-2241. 10.1038/leu.2009.175

Balkwill F. 2004. The significance of cancer cell expression of the chemokine receptor CXCR4. Seminars in Cancer Biology 14:171-179.

Braun M, Qorraj M, Büttner M, Klein FA, Saul D, Aigner M, Huber W, Mackensen A, Jitschin R, and Mougiakakos D. 2016. CXCL12 promotes glycolytic reprogramming in acute myeloid leukemia cells via the CXCR4/mTOR axis. Leukemia 30:1788-1792. 10.1038/leu.2016.58

Burger JA, and Bürkle A. 2007. The CXCR4 chemokine receptor in acute and chronic leukaemia: a marrow homing receptor and potential therapeutic target. British Journal of Haematology 137:288-296.

Crazzolara R, Kreczy A, Mann G, Heitger A, Eibl G, Fink FM, Möhle R, and Meister B. 2001. High expression of the chemokine receptor CXCR4 predicts extramedullary organ infiltration in childhood acute lymphoblastic leukaemia. British Journal of Haematology 115:545-553.

Döhner H, Estey E, Grimwade D, Amadori S, Appelbaum FR, Büchner T, Dombret H, Ebert BL, Fenaux P, Larson RA, Levine RL, Lo-Coco F, Naoe T, Niederwieser D, Ossenkoppele GJ, Sanz M, Sierra J, Tallman MS, Tien H-F, Wei AH, Löwenberg B, and Bloomfield CD. 2017. Diagnosis and management of AML in adults: 2017 ELN recommendations from an international expert panel. Blood 129:424-447. 10.1182/blood-2016-08-733196 
352 Döhner H, Weisdorf DJ, and Bloomfield CD. 2015. Acute Myeloid Leukemia. The New England

353

354

355

356

357

358

359

360

361

362

363

364

365

366

367

368

369

370

371

372

373

374

375

376

377

378

379

380

381

382

383

384

385

386

387

388

389

390

391

392 journal of medicine 373:1136-1152. 10.1056/NEJMra1406184

Grove CS, and Vassiliou GS. 2014. Acute myeloid leukaemia: a paradigm for the clonal evolution of cancer? Disease Models \& Mechanisms 7:941-951. 10.1242/dmm.015974

Janssens R, Struyf S, and Proost P. 2018. Pathological roles of the homeostatic chemokine CXCL12. Cytokine and Growth Factor Reviews 44:51-68. 10.1016/j.cytogfr.2018.10.004

Kell J. 2004. Emerging treatments in acute myeloid leukaemia. Expert opinion on emerging drugs 9:55-71.

Konoplev S, Rassidakis GZ, Estey E, Kantarjian H, Liakou Cl, Huang X, Xiao L, Andreeff M, Konopleva M, and Medeiros LJ. 2007. Overexpression of CXCR4 predicts adverse overall and event-free survival in patients with unmutated FLT3 acute myeloid leukemia with normal karyotype. Cancer 109:1152-1156.

Ladikou EE, Chevassut T, Pepper CJ, and Pepper AG. 2020. Dissecting the role of the CXCL12/CXCR4 axis in acute myeloid leukaemia. British Journal of Haematology 189:815-825. 10.1111/bjh.16456

Mandawat A, Fiskus W, Buckley KM, Robbins K, Rao R, Balusu R, Navenot J-M, Wang Z-X, Ustun C, Chong DG, Atadja P, Fujii N, Peiper SC, and Bhalla K. 2010. Pan-histone deacetylase inhibitor panobinostat depletes CXCR4 levels and signaling and exerts synergistic antimyeloid activity in combination with CXCR4 antagonists. Blood 116:53065315. 10.1182/blood-2010-05-284414

Mantovani A, Savino B, Locati M, Zammataro L, Allavena P, and Bonecchi R. 2010. The chemokine system in cancer biology and therapy. Cytokine and Growth Factor Reviews 21:27-39. 10.1016/j.cytogfr.2009.11.007

Meads MB, Hazlehurst LA, and Dalton WS. 2008. The bone marrow microenvironment as a tumor sanctuary and contributor to drug resistance. Clinical cancer research : an official journal of the American Association for Cancer Research 14:2519-2526. 10.1158/10780432.CCR-07-2223

Meyer SC, and Levine RL. 2014. Translational implications of somatic genomics in acute myeloid leukaemia. The Lancet Oncology 15:e382-e394. 10.1016/S1470-2045(14)70008-7

Möhle R, Failenschmid C, Bautz F, and Kanz L. 1999. Overexpression of the chemokine receptor CXCR4 in B cell chronic lymphocytic leukemia is associated with increased functional response to stromal cell-derived factor-1 (SDF-1). Leukemia 13:1954-1959.

Mrózek K, Heerema NA, and Bloomfield CD. 2004. Cytogenetics in acute leukemia. Blood Reviews 18:115-136.

Nervi B, Ramirez P, Rettig MP, Uy GL, Holt MS, Ritchey JK, Prior JL, Piwnica-Worms D, Bridger G, Ley TJ, and DiPersio JF. 2009. Chemosensitization of acute myeloid leukemia (AML) following mobilization by the CXCR4 antagonist AMD3100. Blood 113:6206-6214. 10.1182/blood-2008-06-162123

Newman AM, Liu CL, Green MR, Gentles AJ, Feng W, Xu Y, Hoang CD, Diehn M, and Alizadeh AA. 2015. Robust enumeration of cell subsets from tissue expression profiles. Nature methods 12:453-457. 10.1038/nmeth.3337 
393

394

395

396

397

398

399

400

401

402

403

404

405

406

407

408

409

410

411

412

413

414

415

416

417

418

419

420

421

422

423

424

425

426

427

Pan J, Mestas J, Burdick MD, Phillips RJ, Thomas GV, Reckamp K, Belperio JA, and Strieter RM. 2006. Stromal derived factor-1 (SDF-1/CXCL12) and CXCR4 in renal cell carcinoma metastasis. Molecular Cancer 5:56.

Petit I, Szyper-Kravitz M, Nagler A, Lahav M, Peled A, Habler L, Ponomaryov T, Taichman RS, Arenzana-Seisdedos F, Fujii N, Sandbank J, Zipori D, and Lapidot T. 2002. G-CSF induces stem cell mobilization by decreasing bone marrow SDF-1 and up-regulating CXCR4. Nature Immunology 3:687-694.

Phillips RJ, Burdick MD, Lutz M, Belperio JA, Keane MP, and Strieter RM. 2003. The stromal derived factor-1/CXCL12-CXC chemokine receptor 4 biological axis in non-small cell lung cancer metastases. American Journal of Respiratory and Critical Care Medicine 167:1676-1686.

Prada-Arismendy J, Arroyave JC, and Röthlisberger S. 2017. Molecular biomarkers in acute myeloid leukemia. Blood Reviews 31:63-76. 10.1016/j.blre.2016.08.005

Rombouts EJC, Pavic B, Löwenberg B, and Ploemacher RE. 2004. Relation between CXCR-4 expression, Flt3 mutations, and unfavorable prognosis of adult acute myeloid leukemia. Blood 104:550-557.

Spoo AC, Lübbert M, Wierda WG, and Burger JA. 2007. CXCR4 is a prognostic marker in acute myelogenous leukemia. Blood 109:786-791.

Taichman RS, Cooper C, Keller ET, Pienta KJ, Taichman NS, and McCauley LK. 2002. Use of the stromal cell-derived factor-1/CXCR4 pathway in prostate cancer metastasis to bone. Cancer Research 62:1832-1837.

van Galen P, Hovestadt V, Wadsworth li MH, Hughes TK, Griffin GK, Battaglia S, Verga JA, Stephansky J, Pastika TJ, Lombardi Story J, Pinkus GS, Pozdnyakova O, Galinsky I, Stone RM, Graubert TA, Shalek AK, Aster JC, Lane AA, and Bernstein BE. 2019. SingleCell RNA-Seq Reveals AML Hierarchies Relevant to Disease Progression and Immunity. Cell 176. 10.1016/j.cell.2019.01.031

Vander Heiden MG, Cantley LC, and Thompson CB. 2009. Understanding the Warburg effect: the metabolic requirements of cell proliferation. Science (New York, NY) 324:1029-1033. 10.1126/science.1160809

Xu Z-J, Ma J-C, Zhou J-D, Wen X-M, Yao D-M, Zhang W, Ji R-B, Wu D-H, Tang L-J, Deng Z-Q, Qian J, and Lin J. 2019. Reduced protocadherin17 expression in leukemia stem cells: the clinical and biological effect in acute myeloid leukemia. Journal of Translational Medicine 17:102. 10.1186/s12967-019-1851-1 


\section{Figure 1}

Expression heat map of differentially expressed cytokine and cytokine receptor genes between AML patients and normal controls.

(A) Differentially expressed genes between AML patients and normal controls in GSE30029.

(B) Differentially expressed genes between AML patients and normal controls in GSE63270.

Only the top 50 genes were shown if adjusted $p$-value $<0.05$ and $|\log \mathrm{FC}|>0$.

A gse30029

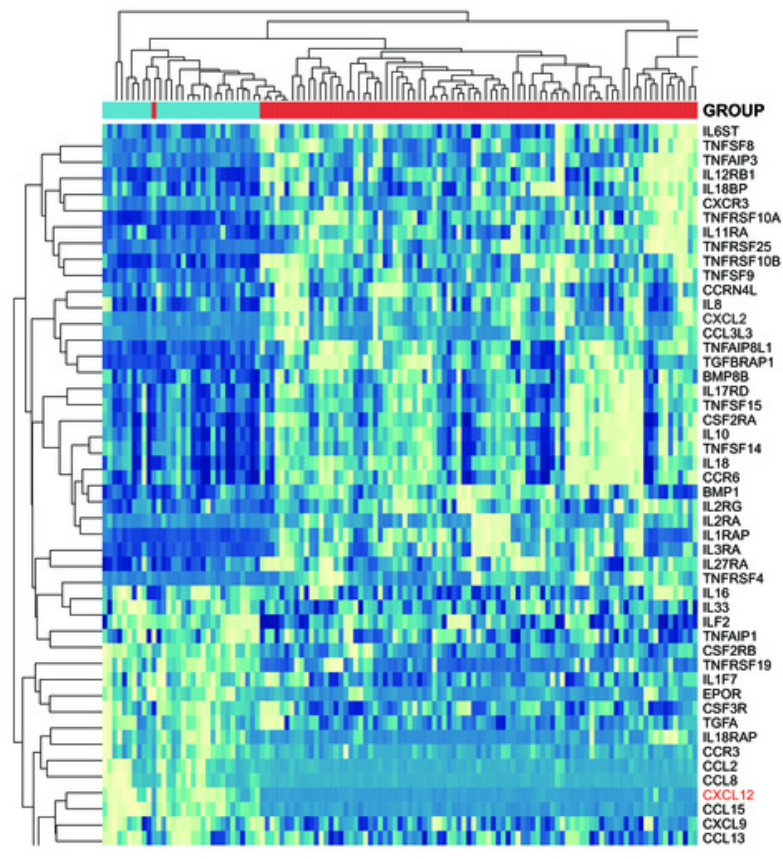

B GSE63270

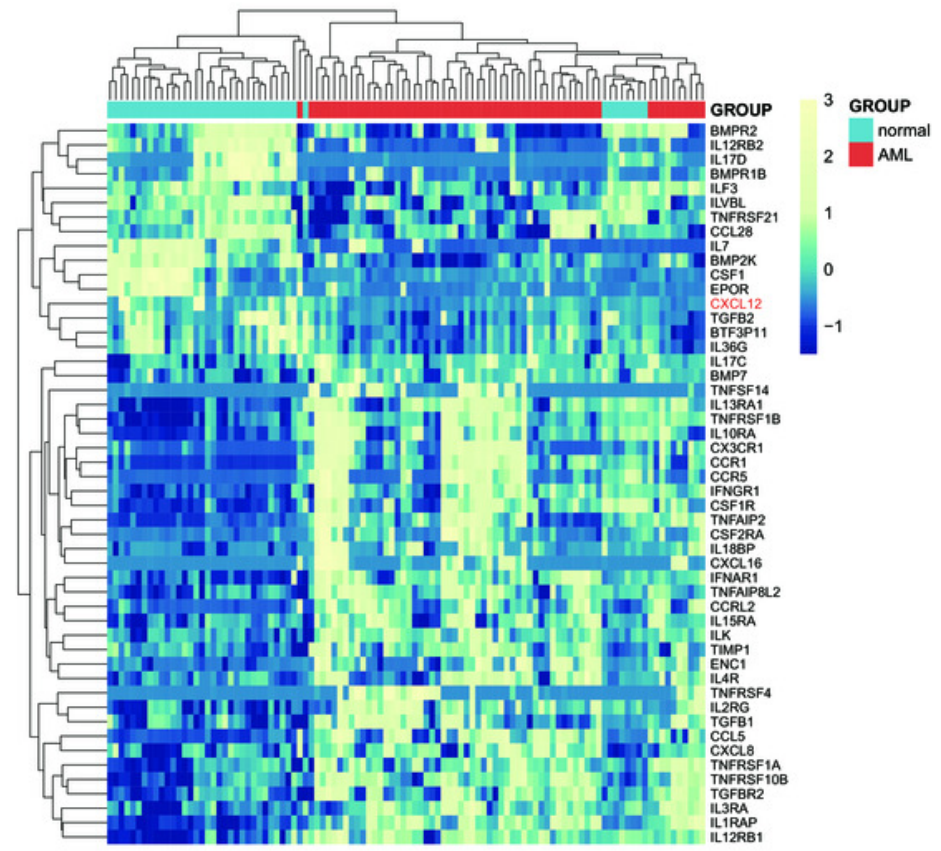


Figure 2

mRNA expression levels of CXCL12 from different tissue origins and tumors.

(A) mRNA expression levels of CXCL12 in different tissues from the GTEx database. (B) mRNA expression levels of CXCL12 in various tumor cell lines from the CCLE database.

A GTEx

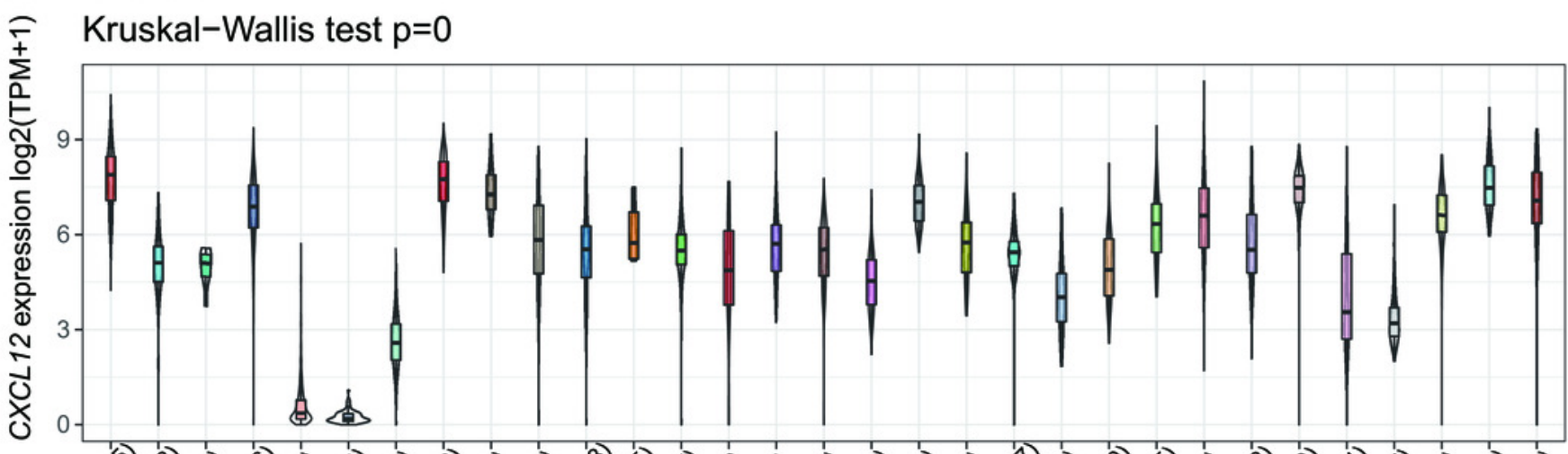

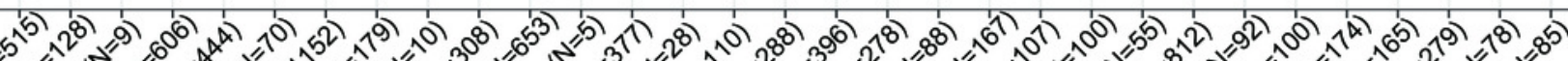
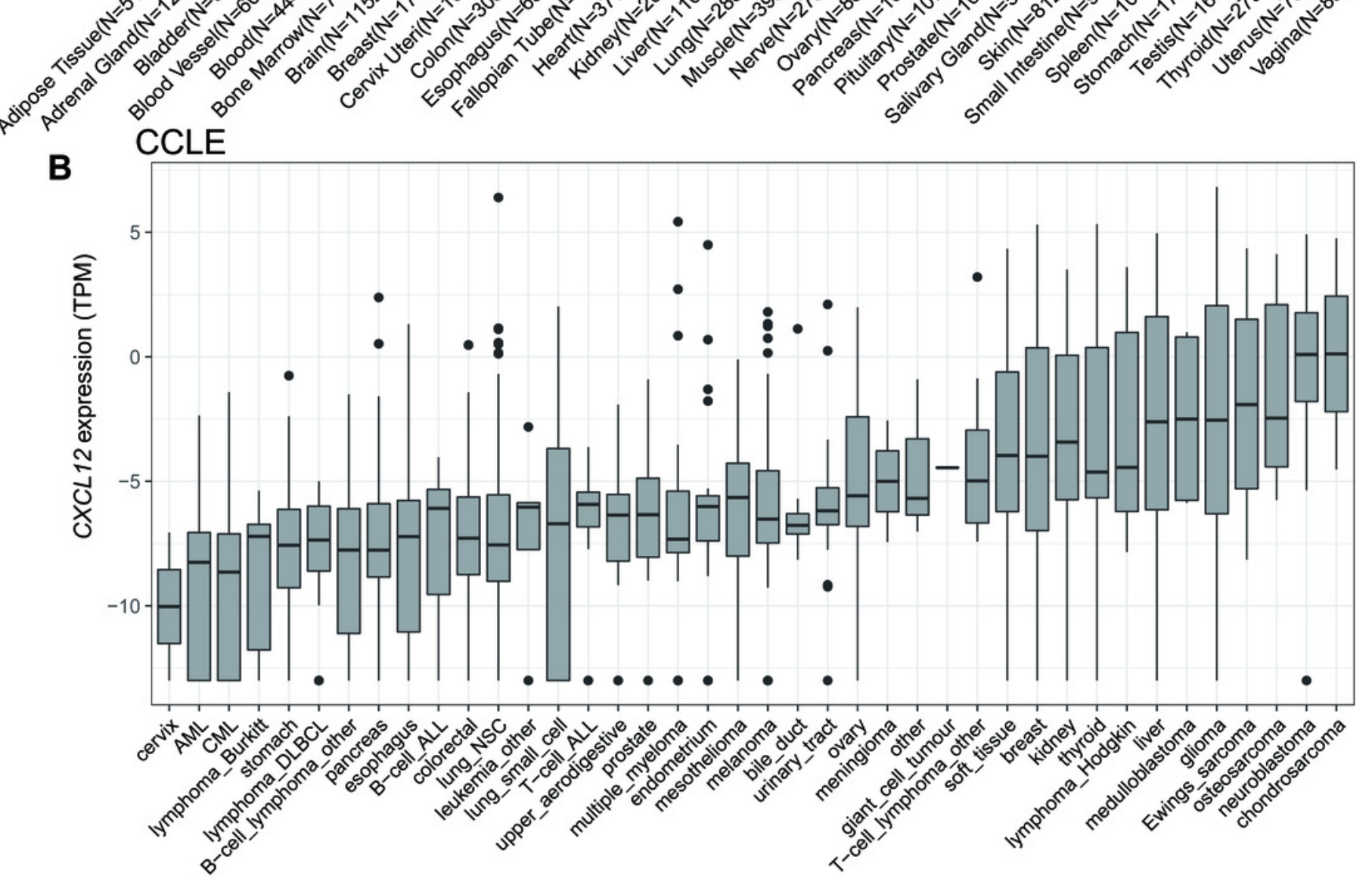
Figure 3

CXCL12 expression in normal hematopoiesis and AML cells.

The CXCL12 expression level of normal controls and AML patients from GSE30029 (A) and GSE63270 (B) datasets.
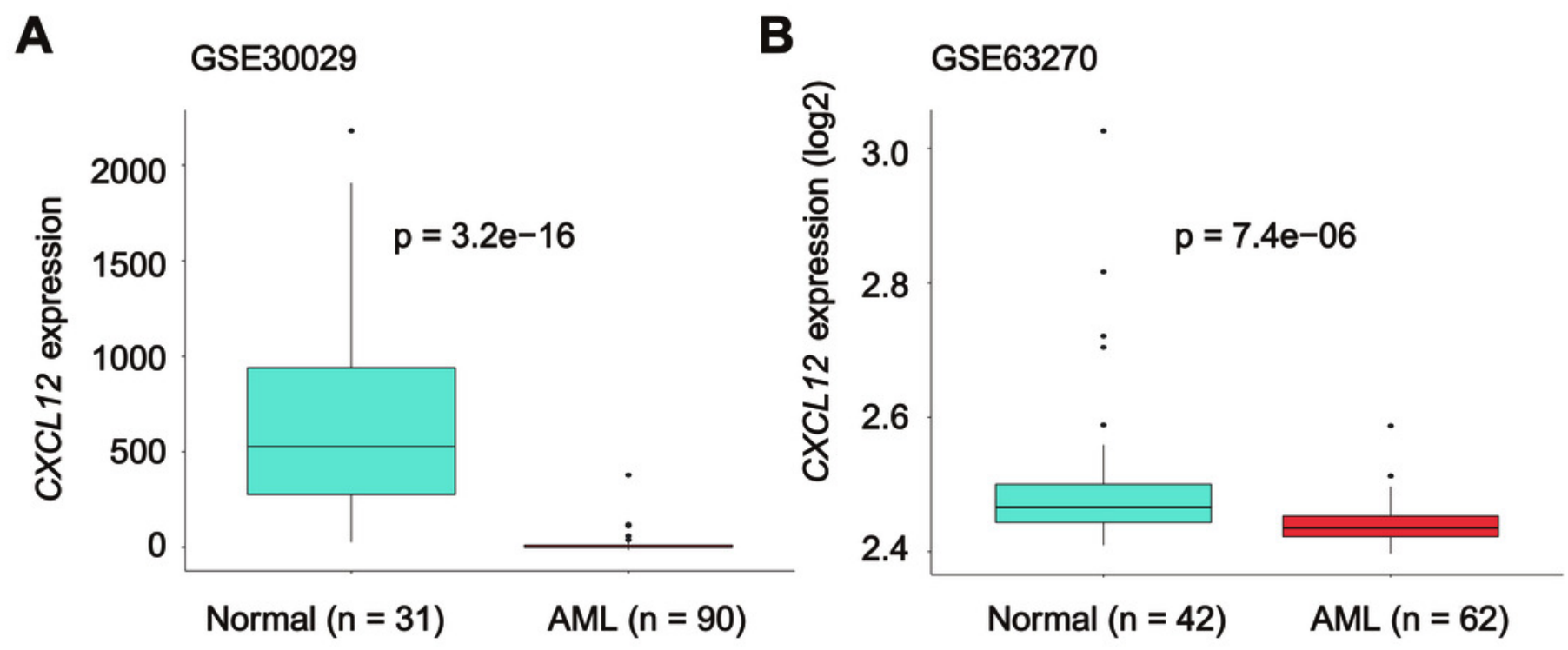
Figure 4

Survival analysis of CXCL12 using the TCGA cohort.

(A, D) The correlation of CXCL12 expression level with OS (A) and EFS (D) for the total AML patients. (B, E) The correlation of CXCL12 expression level with OS (B) and EFS (E) for the non-M3-AML patients. (C, F) The correlation of CXCL12 expression level with OS (C) and EFS (F) for the CN-AML patients.

A

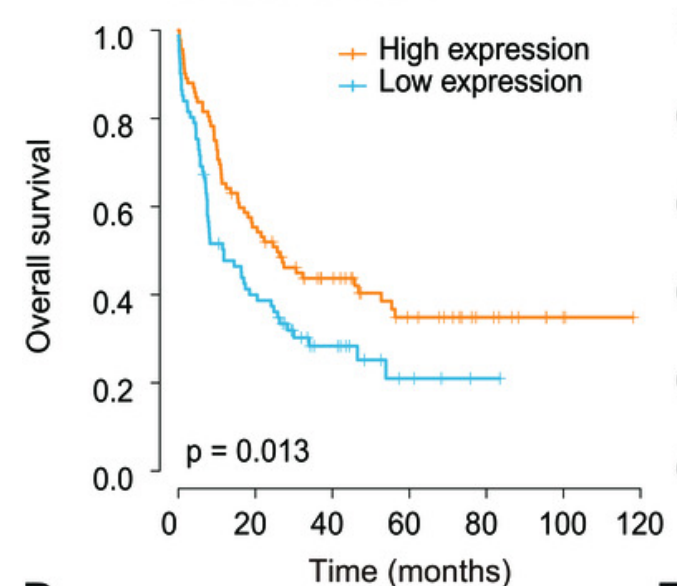

D Whole-AML TCGA

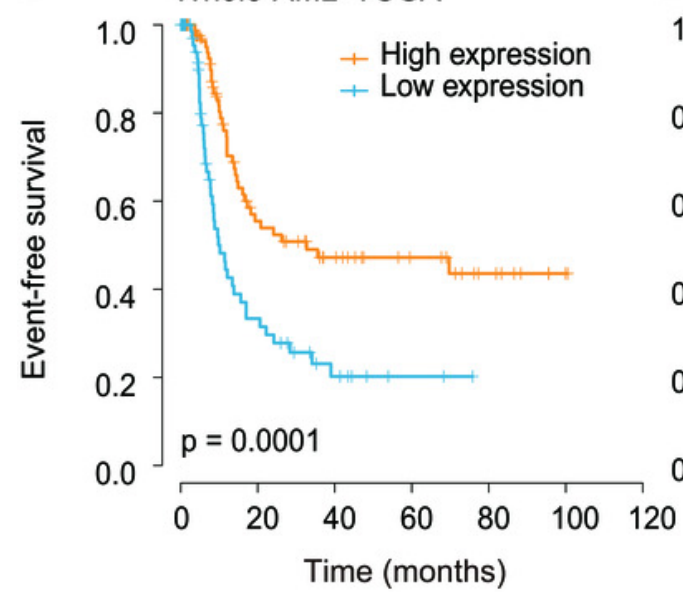

E
B non-M3-AML TCGA

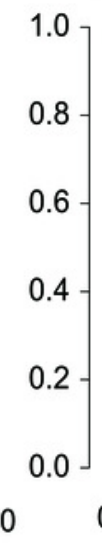

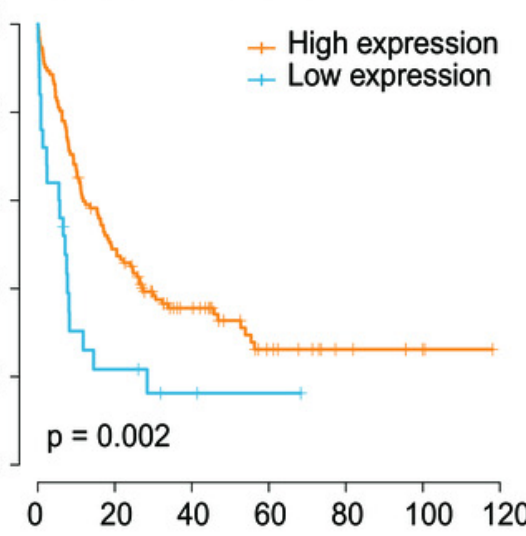

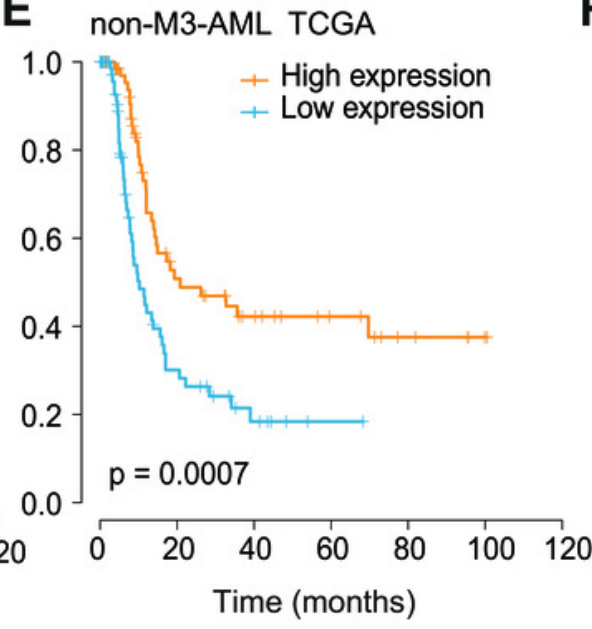

C CN-AML TCGA

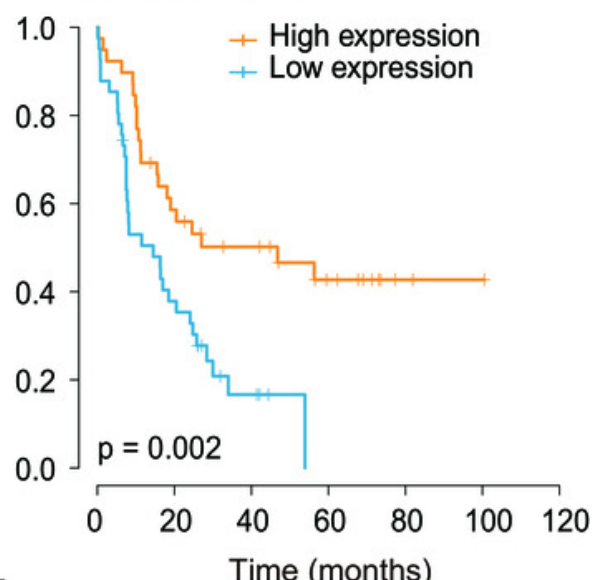

$\mathbf{F}$

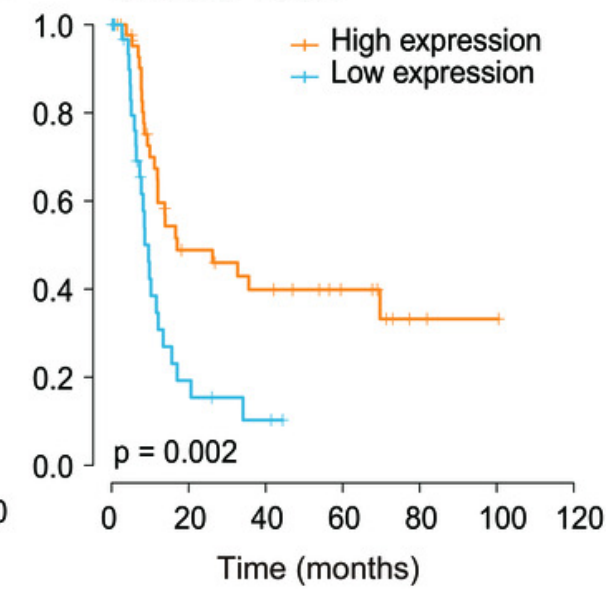


Figure 5

Prognostic significance of CXCL12 in the molecularly defined subgroups in the TCGA cohort.

(A) Kaplan-Meier estimate for OS of FLT3-ITD-negative AML patients. (B) Kaplan-Meier estimate for OS of NPM1-mutated AML patients.

A

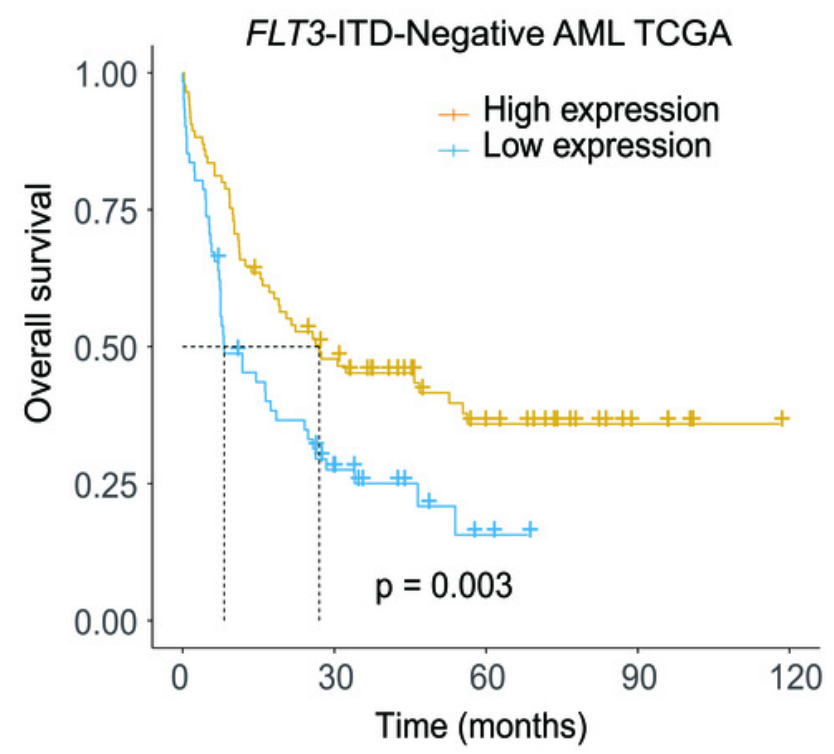

B

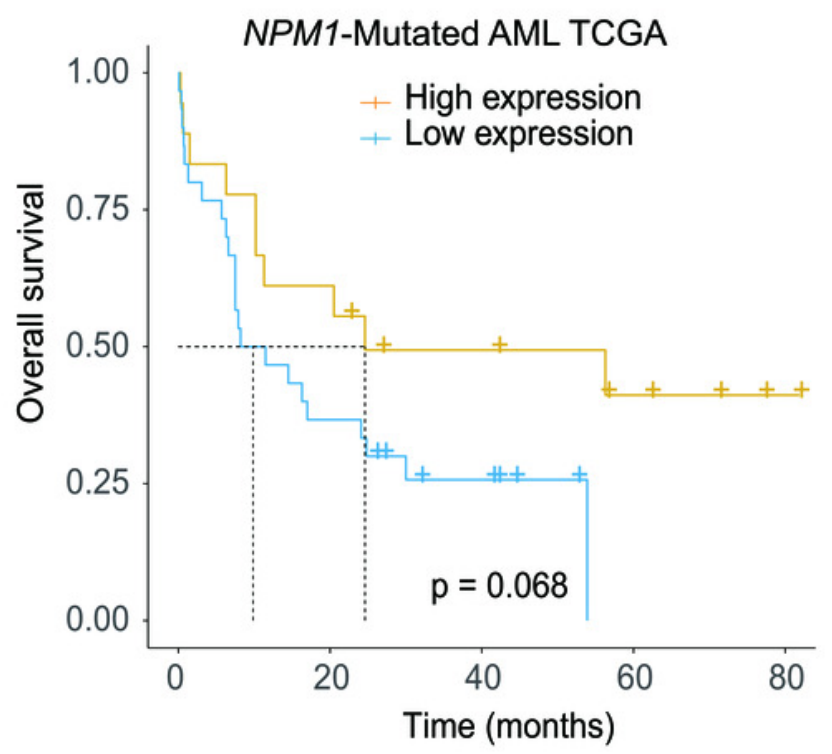


Figure 6

Immune cell infiltration difference between high and low CXCL12 expressers.

The Violin plot exhibits the difference between 22 CIBERSORT immune cell fractions between high and low CXCL12 expressers.

Hgh CXCL12 国 Low CXCL12

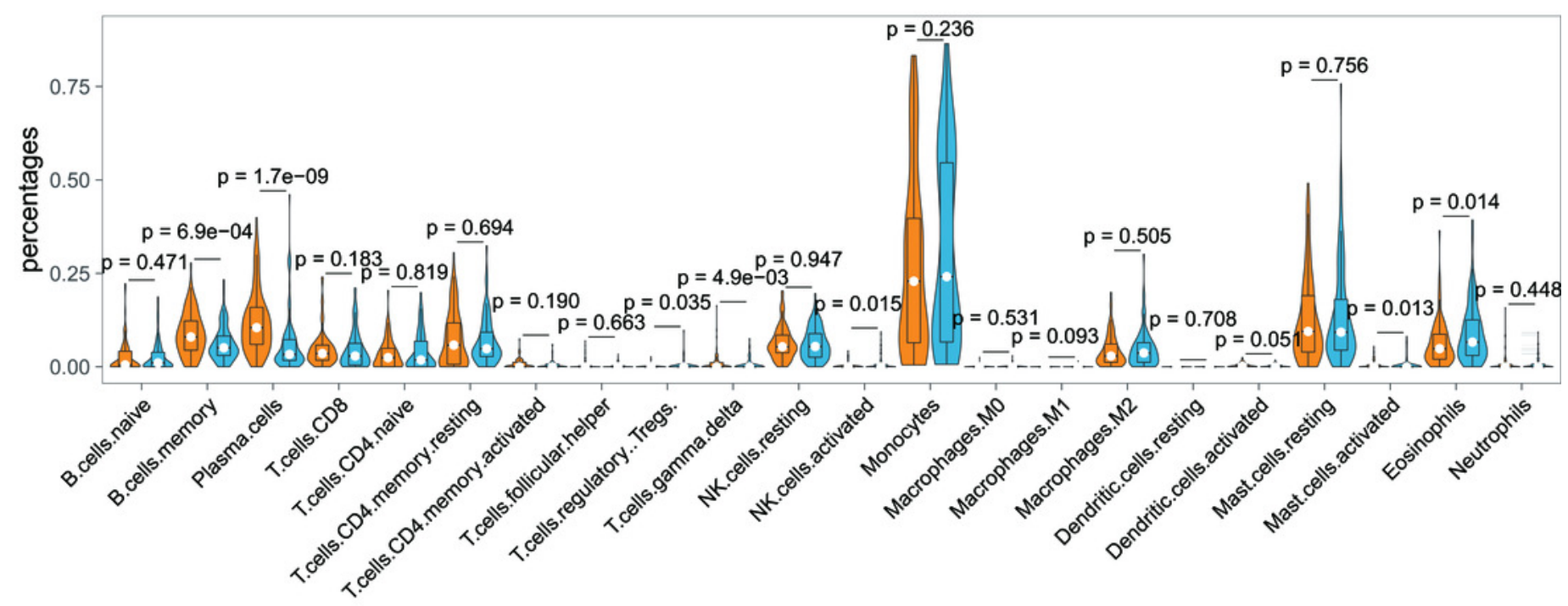




\section{Figure 7}

GO and KEGG pathways analysis of differentially expressed genes between high and low CXCL12 expressers.

The top-ten statistically significant results identified including Biological Process (BP), Cellular Component (CC), Molecular Function (MF), and KEGG Pathways are listed according to their $\log 10$ ( $p$-value) (colored bars).

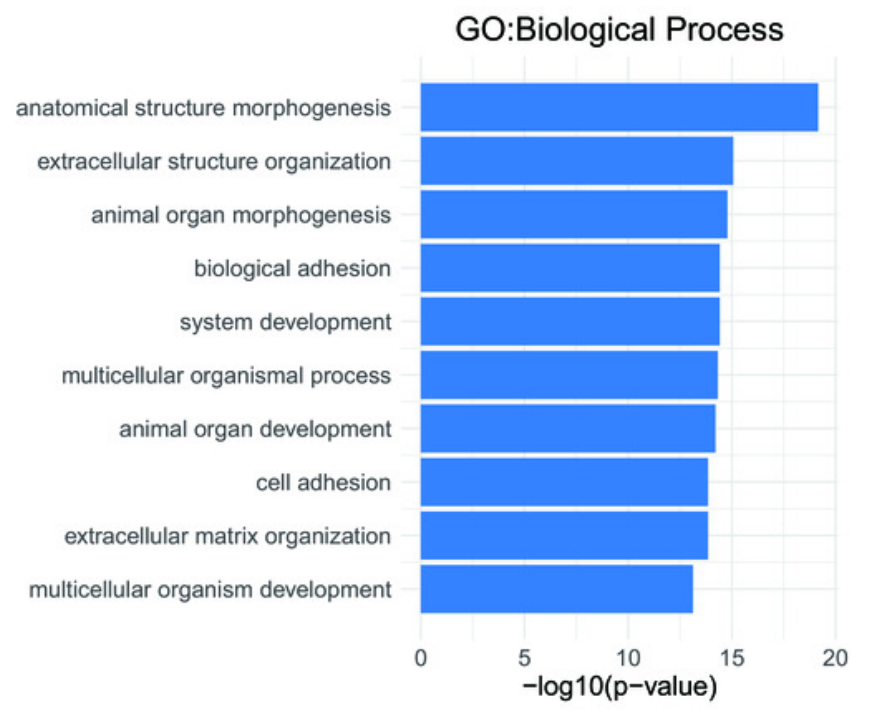

GO:Molecular Function

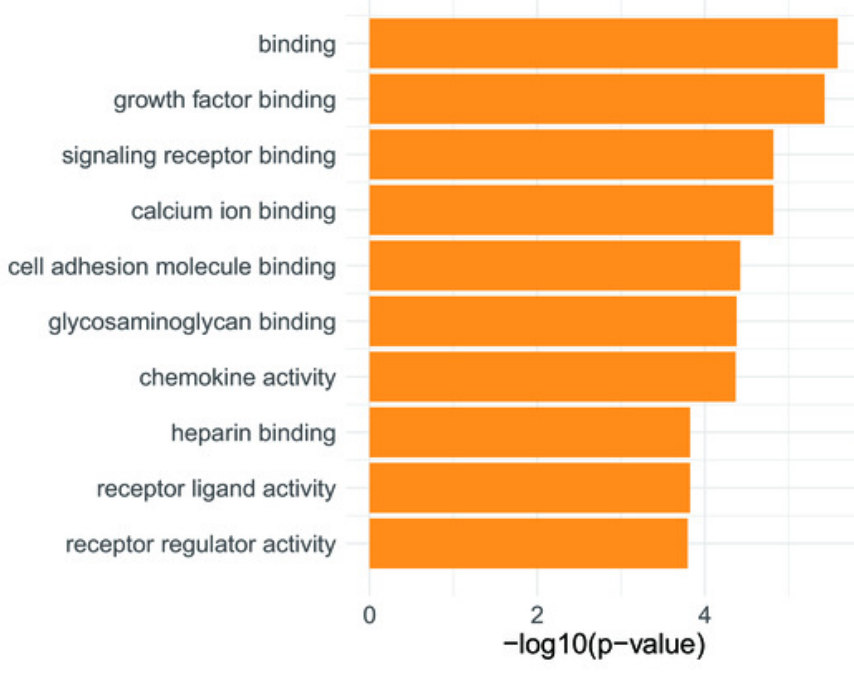

GO:Cellular Component

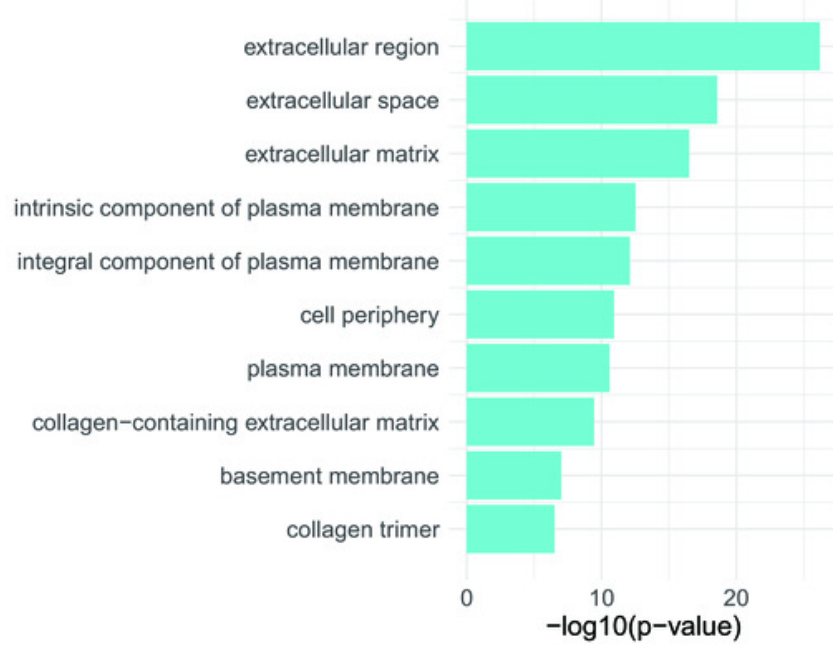

KEGG Pathways

Protein digestion and absorption ECM-receptor interaction Focal adhesion PI3K-Akt signaling pathway Cytokine-cytokine receptor interaction Relaxin signaling pathway Neuroactive ligand-receptor interaction Chemokine signaling pathway Renin secretion

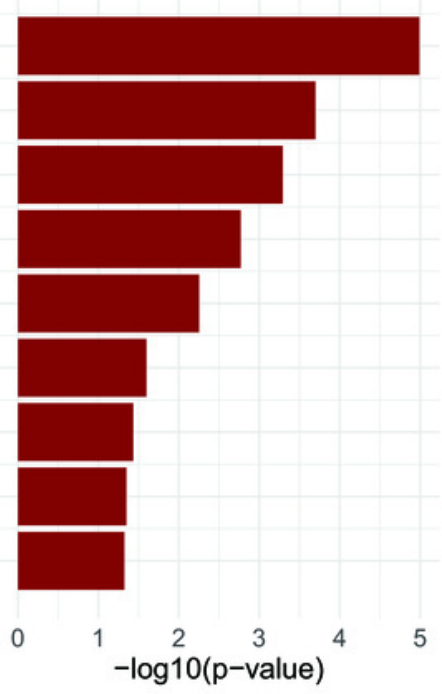




\section{Table 1 (on next page)}

Patient characteristics with respect to CXCL12 expression.

Abbreviations: AML, acute myeloid leukemia; TCGA, The Cancer Genome Atlas; WBC, white blood cells; BM, bone marrow; PB, peripheral blood; ITD, internal tandem duplication. 


\begin{tabular}{|c|c|c|c|}
\hline \multirow{2}{*}{$\begin{array}{l}\text { Patient } \\
\text { characteristics }\end{array}$} & \multicolumn{3}{|c|}{ AML (TCGA dataset) } \\
\hline & $\begin{array}{l}\text { High } C X C L 12 \\
\quad(\mathrm{n}=86)\end{array}$ & $\begin{array}{c}\text { Low } C X C L 12 \\
(n=87)\end{array}$ & $\boldsymbol{P}$ \\
\hline Age, years & & & 0.537 \\
\hline Median & 60 & 57 & \\
\hline Range & $21-88$ & $18-81$ & \\
\hline Sex (male/female) & $52 / 34$ & $40 / 47$ & 0.056 \\
\hline WBC count, $\times 10^{9} / \mathrm{L}$ & & & $<0.0001$ \\
\hline Median & 6.4 & 42.1 & \\
\hline Range & $0.4-116.2$ & $0.9-297.4$ & \\
\hline BM blasts, $\%$ & & & $<0.001$ \\
\hline Median & 62 & 77 & \\
\hline Range & $30-100$ & $32-100$ & \\
\hline PB blasts, $\%$ & & & $<0.0001$ \\
\hline Median & 14 & 52 & \\
\hline Range & $0-97$ & $0-98$ & \\
\hline Karyotype & & & 0.872 \\
\hline Favorable & 15 & 17 & \\
\hline Intermediate & 50 & 51 & \\
\hline Adverse & 20 & 17 & \\
\hline Unknown & 1 & 2 & \\
\hline FLT3-ITD & & & 0.010 \\
\hline Present & 7 & 19 & \\
\hline Absent & 79 & 66 & \\
\hline$N P M 1$ & & & 0.015 \\
\hline Mutated & 17 & 31 & \\
\hline Wild-type & 69 & 54 & \\
\hline CEBPA & & & 0.318 \\
\hline Single mutated & 3 & 5 & \\
\hline Double mutated & 4 & 1 & \\
\hline Wild-type & 79 & 79 & \\
\hline IDH1 & & & 0.980 \\
\hline Mutated & 8 & 8 & \\
\hline Wild-type & 78 & 77 & \\
\hline$I D H 2$ & & & 0.818 \\
\hline Mutated & 9 & 8 & \\
\hline Wild-type & 77 & 77 & \\
\hline$R U N X 1$ & & & 0.062 \\
\hline Mutated & 11 & 4 & \\
\hline Wild-type & 75 & 81 & \\
\hline
\end{tabular}




\begin{tabular}{lccc}
$\begin{array}{l}\text { DNMT3A } \\
\text { Mutated }\end{array}$ & 21 & 21 & 0.965 \\
Wild-type & 65 & 64 & \\
\hline Table continued & & & \\
\hline Patient & & AML (TCGA dataset) & \\
characteristics & High $\boldsymbol{C X C L 1 2}$ & Low $\boldsymbol{C X C L 1 2}$ & $\boldsymbol{P}$ \\
& $(\mathbf{n}=\mathbf{8 6})$ & $(\mathbf{n}=\mathbf{8 7})$ & 0.046 \\
\hline TP53 & 10 & 3 & \\
Mutated & 76 & 82 & \\
Wild-type & & &
\end{tabular}

1 


\section{Table 2 (on next page)}

Multivariate analysis of CXCL12 expression for overall survival in the TCGA cohorts.

Abbreviations: TCGA, The Cancer Genome Atlas; CN-AML, cytogenetically normal AML; WBC, white blood cells; $\mathrm{Cl}$, confidence interval.

NOTE: Hazard Ratio $>1$ or Hazard Ratio $<1$ indicate a higher or lower risk. Only variables with a univariable $P \leq 0.20$ were included in the multivariable models.

${ }^{a}$ Low vs high expression.

${ }^{b}>60$ vs $\leq 60$ years.

${ }^{\mathrm{c}} \geq 30$ vs $<30 \times 10^{9} / \mathrm{L}$.

${ }^{\mathrm{d}}$ Adverse vs intermediate vs favorable.

${ }^{\mathrm{e}}$ Mutated vs wild type. 


\begin{tabular}{lcccc}
\hline & \multicolumn{2}{c}{ Whole-AML $(\mathbf{n}=\mathbf{1 7 3})$} & \multicolumn{2}{c}{ CN-AML $(\mathbf{n}=\mathbf{8 0})$} \\
\cline { 2 - 5 } Variables & $\begin{array}{c}\text { Hazard Ratio } \\
(\mathbf{9 5 \%} \mathbf{C I})\end{array}$ & $\boldsymbol{P}$ & $\begin{array}{c}\text { Hazard Ratio } \\
\mathbf{( 9 5 \%} \mathbf{~ C I})\end{array}$ & $\boldsymbol{P}$ \\
\hline$C X C L 12^{\mathrm{a}}$ & $2.25(1.51-3.35)$ & $<0.0001$ & $2.14(1.15-3.97)$ & 0.016 \\
Age $^{\mathrm{b}}$ & $1.01(1.00-1.03)$ & $<0.0001$ & $2.37(1.35-4.18)$ & 0.003 \\
WBC count $^{\mathrm{c}}$ & $3.14(1.92-5.14)$ & $<0.0001$ & $1.21(0.67-2.19)$ & 0.520 \\
Cytogenetic risk $^{\mathrm{d}}$ & $1.79(1.25-2.57)$ & 0.002 & - & - \\
$T P 53^{\mathrm{e}}$ & $2.85(1.41-5.75)$ & 0.004 & - & - \\
$R U N X 1^{\mathrm{e}}$ & $2.08(1.10-3.93)$ & 0.023 & - & - \\
$D N M T 3 A^{\mathrm{e}}$ & $1.74(1.13-2.68)$ & 0.012 & $1.95(1.11-3.44)$ & 0.020 \\
$I D H 1^{\mathrm{e}}$ & - & - & $0.60(0.21-1.75)$ & 0.351 \\
\hline
\end{tabular}

1 